\title{
The Finance-Investment-Growth Causal Connection: Evidence from the Republic of Congo
}

\author{
Brian Muyambiri ${ }^{1 *}$
}

${ }^{1}$ Botswana Open University, BOTSWANA

*Corresponding Author: bmuyambiri@staff.bou.ac.bw

Citation: Muyambiri, B. (2020). The Finance-Investment-Growth Causal Connection: Evidence from the Republic of Congo. Dutch Journal of Finance and Management, 4(1), em0061. https://doi.org/10.29333/djfm/8364

\section{ARTICLE INFO}

Received: 12 May 2020

Accepted: 15 Jun. 2020

\begin{abstract}
This paper examines the causal relationship between financial development and investment in the Republic of Congo for the period 1960 to 2017. The causal relationship between financial development, investment and economic growth is examined using four multivariate Granger-causality models. The study found that the finance-investment-growth causal relationship is highly dependent on the choice of the financial development indicator used. In addition, all financial development indicators, except domestic credit provided to the private sector, were found to Granger cause investment in the short run. Furthermore, investment has been found to Granger cause economic growth in the short run when domestic credit provided by the financial sector and the composite financial development index are included as measures of financial development in subsequent models. Therefore, dependent on the policy focus, economic policy through financial development should take into account the financial indicator so as to predict and/or achieve the resultant policy objectives.
\end{abstract}

Keywords: financial development, investment, multivariate granger causality, Republic of Congo

\section{INTRODUCTION}

The causal relationship between financial development and investment has been assumed in economic circles as a given. The preponderant view has been the notion that finance enhances investment through its relationship with economic growth. Foremost, among others, in this presupposition are the works of McKinnon (1973), Shaw (1973) and Levine (1997, 2005). However, a look at related literature (on the financeinvestment causal relationship) shows that the aforementioned presupposition, though popular in econometric circles, is not the only outcome when an empirical investigation is done.

It is against this background and the absence of such a finance-investment-growth study on a developing and low middle-income country like the Republic of Congo is this article commissioned to clearly ascertain the extent of the finance-investment-growth causal connection. Previous studies, on the same topic, have focused on panel data and cross-sectional studies that do not specifically address the country-specific effects that underlie the relationship between finance and investment. More so, most studies on this subject focus on mostly developed and at least middle or higherincome developing countries. Not to mention the incessant use of bivariate causality models and their associated weakness of the omission of variable bias. Therefore, to address the aforementioned knowledge and methodological gaps, this study examines the causal relationship between financial development, investment and economic growth in the Republic of Congo using time series analysis. It employs the autoregressive distributed lag (ARDL) bounds method in a multivariate setting to evaluate the stipulated causal relationship. The ARDL bounds approach is preferred because it is more efficient than the usual residual-based error correction techniques, it can be used even when variables are integrated to a maximum order of 1 and it has superior small sample estimation properties than the usual preferred residual-based methods.

The remainder of the paper is organised as follows: firstly, an initial discussion of Congo's finance-investment-growth dynamics; followed by, a presentation of the data sources, the empirical model specifications and the estimation technique; the empirical results; and finally, the conclusion of the study.

\section{FINANCE-INVESTMENT-GROWTH DYNAMICS IN THE CONGO}

The Republic of Congo is a country in Central Africa bordered by five countries i.e. Gabon, Cameroon, the Central African Republic, the Democratic Republic of the Congo and 
30

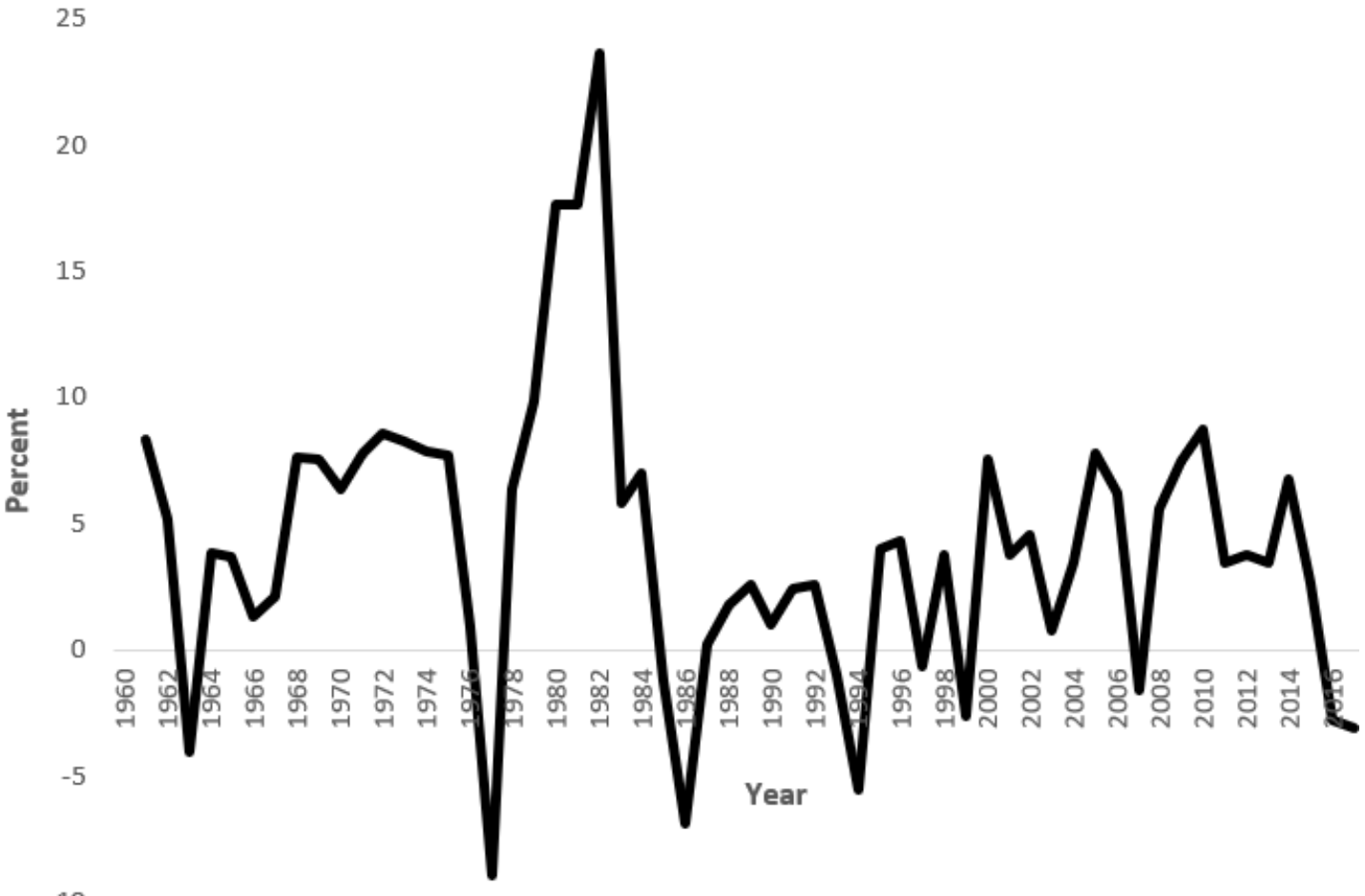

$-10$

$-15$

Figure 1. Trends in GDP growth (1960-2017)

Source: World Bank, World Development Indicators, 2019

the Angolan Cabinda. It gained its independence from the French in 1960 (Decalo, 1990). However, the Congo, like all other central African countries has had its full share of political instability with a number of changes in the country's leadership being replaced through coups since its independence (Clark, 2005). Nevertheless, the financeinvestment-growth dynamics, since its independence, have continued to show some degree of normalcy.

For example, a look at the trends in GDP growth rates from the time of Congo's independence to now shows that it has experienced relatively above-average years of positive growth than negative growth. Trends in GDP growth are shown in Figure 1.

The first decrease in GDP in the Congo was experienced in 1963, that is, negative $4 \%$. This might have been due to political instability brought about by the ousting of the then president of the country (Decalo, 1990). From then on, the Congo experienced some positive economic growth (averaging $4 \%)$ until 1977. In 1977, the economic growth rate decreased to its lowest ever - a negative $9 \%$ growth. This may be due to the inherent political instability that resulted from the assassination of the then president (President Ngouabi). President Ngouabi had come into power through a coup in 1968 (Decalo, 1990). President Nguesso took over only to lose his power through elections in 1992 (Clark, 2002). However, after the 1997 civil war, he took over the presidency and has been ruling the country ever since. Despite all these happenings, the economy of the Congo appears to have weathered the storm and continued to show a steady average growth rate through the whole period averaging $4 \%$ for the whole period spanning 1960 to 2017.

On the financial front, the Republic of Congo is only made up of the banking sector. There is no stock exchange in the Republic. Congo's financial system is made up of eleven commercial banks that mostly offer corporate banking services and work closely with the Bank of the Central African States (BEAC) which is headquartered in Cameroon. The BEAC serves as the central bank for six central African countries, that is, Cameroon, Central African Republic, Chad, Equatorial Guinea, Gabon and the Republic of the Congo (BEAC, 2019). These countries make up the Economic and Monetary Community of Central Africa. However, overall authority for the country's banking system rests with the Ministry of Finance. Trends in the financial sector of the Congo are shown in Figure 2.

Ever since the Congo's independence to the year 2005, the percentage of liquid liabilities to GDP has been relatively stable ranging from $23 \%$ (just after independence) to $12 \%$ (in the early 2000). After 2005, liquid liabilities have been on an upward trend which saw them reaching an apex of 44\% of GDP in 2015 only to decline to the above average level of $33 \%$ by 2017. The increased liquid liabilities show an increase in the importance of the banking sector in the economy. It translates to an increase in financial development evidenced by more money balances being help by the banking sector. Relatively, 
50

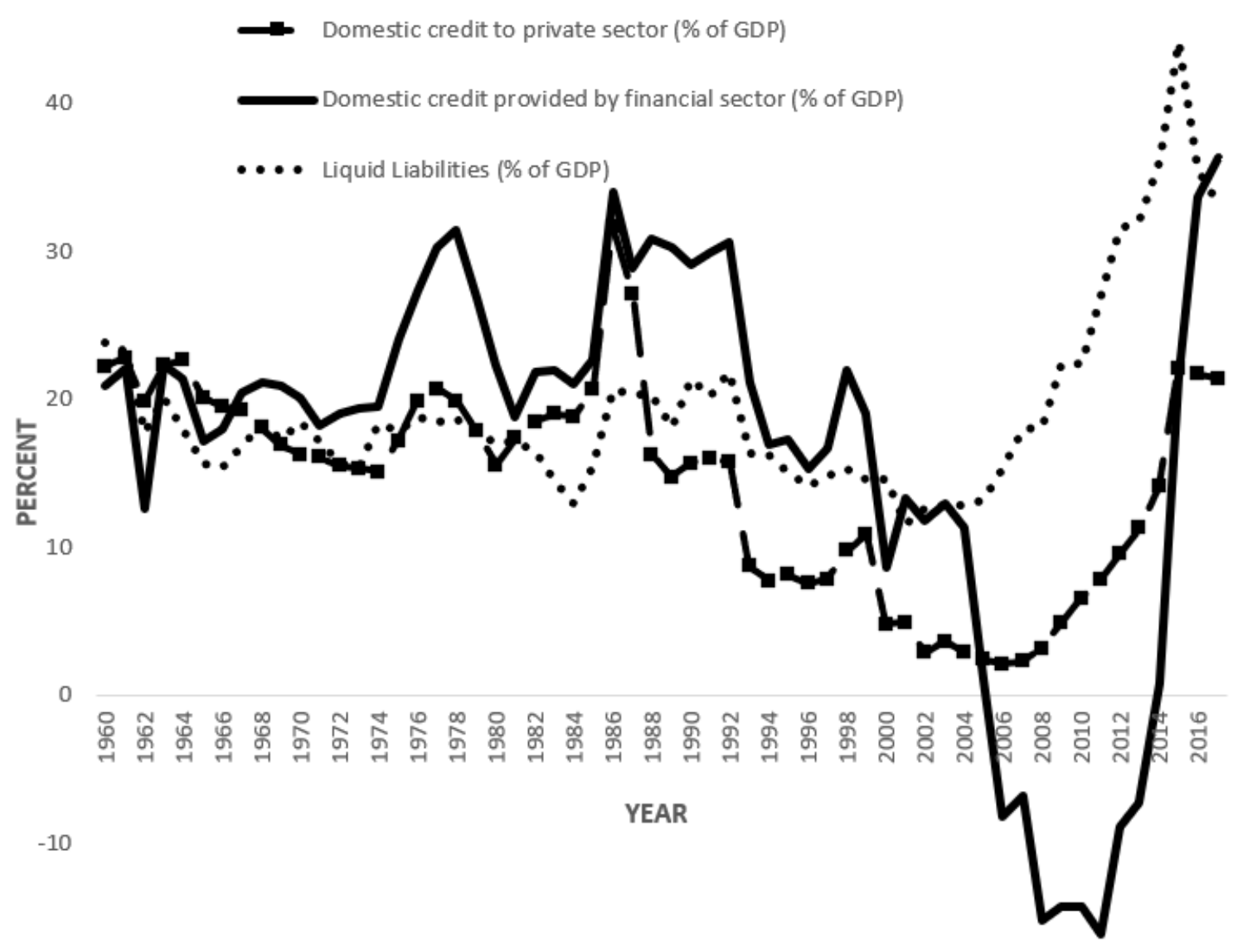

$-20$

Figure 2. Trends in Financial development Indicators (1960-2017)

Source: World Bank, World Development Indicators, 2019

domestic credit provided by the financial sector started, from 1960 to 1974 and 1981 to 1985 , with an average of $18 \%$ and $21 \%$ of GDP, respectively. There were periods of upsurge in the domestic credit provided by the financial sector from 1975 to 1978 and from 1986 to 1992. After 1992, the domestic credit provided by the financial sector has been on a downward trend moving from $31 \%$ of GDP in 1992 to negative 16\% of GDP by 2011. Only to gradually increase to 36\% of GDP by 2017 . This recent increase in the domestic credit provided by the financial sector is evidence of increased financial development as far as credit provision by the financial sector is concerned in the Congo. The same trends can be deciphered, though at lower but positive percentages to those of the domestic credit provided by the financial sector, for the domestic credit provided to the private sector. Overall, trends show that there has been an increase in financial development, especially after the mid 2000s, in the Congo.

The trends in investment (proxied by the gross fixedcapital formation to GDP ratio) and savings (proxied by gross domestic savings) are shown in Figure 3.

The trends in investment show intermittent changes that kept the range between $65 \%$ and $14 \%$ from 1960 to 2017 . The general trend in investment patterns in the Congo show that though relatively stable, investment levels have decreased marginally from an average of $30 \%$ before 1988 to an average of $27 \%$ after 1988 . Otherwise, gross domestic savings trends show (despite the intermittent upsurges and down surges) a general sustained increase from a negative $12 \%$ in 1960 to $51 \%$ by 2017 . This general increase might explain the increase in liquid liabilities that has been noted earlier. Notable though is the unfolding implication that the increased financial development did not enhance both economic growth and investment. An empirical analysis of the said trends would shed greater light on the implied finance-investment-growth relationship.

\section{LITERATURE REVIEW}

The finance-investment-growth nexus has been investigated alternatingly with many studies focusing on either the relationship between finance and economic growth and others on investment and economic growth. A few studies have assessed the finance-investment nexus. However, most of the studies have focused more on developing nations than on developed nations. And less of them have focused on lower income countries like the Republic of Congo which has been plagued by periods of political instability since its independence.

Nevertheless, as far as the causal relationship of the finance-investment-growth nexus is concerned, deductions 
70

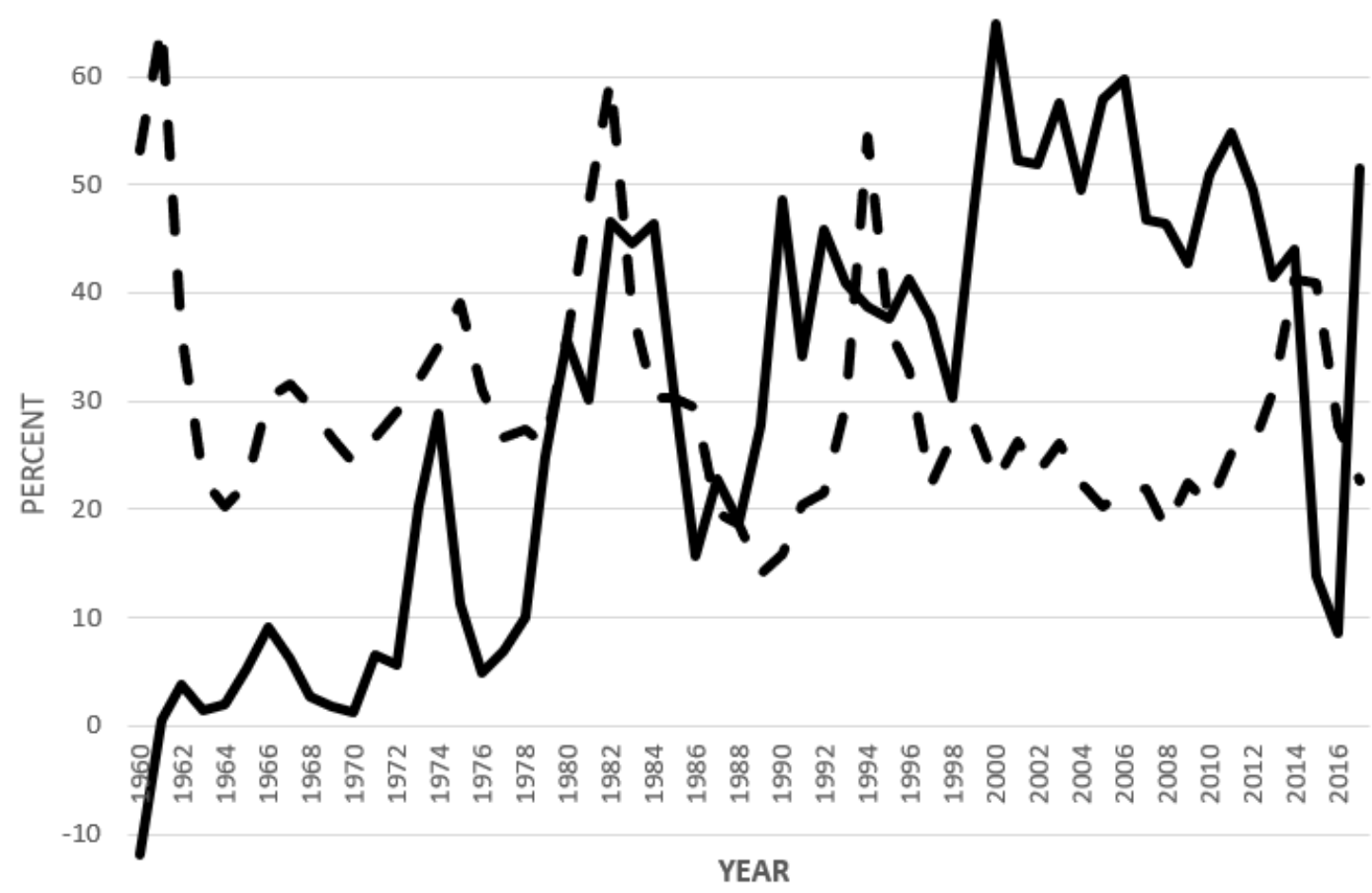

$-20$

\section{- Investment (\% of GDP) Gross domestic savings (\% of GDP)}

Figure 3. Trends in Savings and Investment (1960-2017)

Source: World Bank, World Development Indicators, 2019

have found in general three main results. These can be generally summarized as studies that found bidirectional causality, unidirectional causality and no causality between any two of the variables under discussion.

As far as the finance-growth causality debate is concerned, a number of deductions have been postulated. Studies that found out that financial development Granger causes economic growth include among others Jung (1986), King and Levine (1993a), Odedokun (1996b), Neusser and Kugler (1998), Rousseau and Wachtel (1998), Ghali, (1999), Levine, Loayza and Beck (2000), Beck, Levine and Loayza (2000), Jalilian and Kirkpatrick (2002), Akinlo and Egbetunde (2010), Hussain and Chakraborty (2012) and Nyasha and Odhiambo (2015). On the other hand, studies that found the reverse (economic growth Granger cause financial development) include among others Odhiambo (2004), Ang and McKibbin (2007), Odhiambo (2008b) and Rachdi and Mbarek (2011). Studies that found a biderictional causality between the two include among others Berthelemy and Varoudakis (1996), Luintel and Khan (1999), Fase and Abma (2003) and Abu-Bader and Abu-Qarn (2008).

As far as the finance - investment causal relationship is concerned, the following deductions have been realizedfinancial development Granger-causes investment, investment Granger-causes financial development, bidirectional causality and no causal relationship. Studies that have been for the first deduction include among others $\mathrm{Xu}$, (2000), Caporale, Howells and Soliman (2005), Chaudry (2007), Carp (2012) and Asongu (2014). Odhiambo (2010) validates that investment Granger-causes financial development while
Shan and Jianhong (2006) and Huang (2011) validate the notion of bidirectional causality. Majid (2008) and, Shan and Morris (2002) validate the existence of no causal relationship (Muyambiri and Odhiambo, 2018). As shown by the number of studies reviewed, the aforementioned deductions suggest that the finance-investment-growth relationship is relatively ambiguous, and there is much scope for further empirical investigation.

\section{DATA AND METHODOLOGY}

The study used data for the period of 1960 to 2017. The main data source was the World Development Indicators (World Bank, 2019). The ARDL model used in this study can be expressed as follows:

$$
\begin{aligned}
\Delta I N V_{t}=\propto_{0}+\sum_{i=1}^{n} \propto_{1 i} & \Delta I N V_{t-i}+\sum_{i=0}^{n} \propto_{2 i} \Delta B F A_{t-i} \\
& +\sum_{i=0}^{n} \propto_{3 i} \Delta I N F_{t-i}+\sum_{i=0}^{n} \propto_{4 i} \Delta G D C_{t-i} \\
& +\sum_{i=0}^{n} \propto_{5 i} \Delta G D S_{t-i}+\alpha_{6} I N V_{t-1} \\
& +\alpha_{7} B F A_{t-1}+\alpha_{8} I N F_{t-1}+\alpha_{9} G D C_{t-1} \\
& +\alpha_{10} G D S_{t-1}+\varepsilon_{1 t}
\end{aligned}
$$




$$
\begin{aligned}
& \Delta B F A_{t}=\beta_{0}+\sum_{i=1}^{n} \beta_{1 i} \Delta B F A_{t-i}+\sum_{i=0}^{n} \beta_{2 i} \Delta I N V_{t-i} \\
& +\sum_{i=0}^{n} \beta_{3 i} \Delta I N F_{t-i}+\sum_{i=0}^{n} \beta_{4 i} \Delta G D C_{t-i} \\
& +\sum_{i=0}^{n} \beta_{5 i} \Delta G D S_{t-i}+\beta_{6} B F A_{t-1}+\beta_{7} I N V_{t-1} \\
& \begin{array}{l}
+\beta_{8} I N F_{t-1}+\beta_{9} G D C_{t-1}+\beta_{10} G D S_{t-1} \\
+\varepsilon_{2 t}
\end{array} \\
& \Delta G D S_{t}=\rho_{0}+\sum_{i=1}^{n} \rho_{1 i} \Delta G D S_{t-i}+\sum_{i=0}^{n} \rho_{2 i} \Delta I N V_{t-i} \\
& +\sum_{i=0}^{n} \rho_{3 i} \Delta B F A_{t-i}+\sum_{i=0}^{n} \rho_{4 i} \Delta I N F_{t-i} \\
& +\sum_{i=0}^{n} \rho_{5 i} \Delta G D C_{t-i}+\rho_{6} G D S_{t-1}+\rho_{7} B F A_{t-1} \\
& +\stackrel{i=0}{\rho_{8} I N F_{t-1}+\rho_{9} I N V_{t-1}+\rho_{10} G D C_{t-1}+\varepsilon_{3 t}} \\
& \Delta G D C_{t}=\gamma_{0}+\sum_{i=1}^{n} \gamma_{1 i} \Delta G D C_{t-i}+\sum_{i=0}^{n} \gamma_{2 i} \Delta I N V_{t-i} \\
& +\sum_{i=0}^{n} \gamma_{3 i} \Delta B F A_{t-i}+\sum_{i=0}^{n} \gamma_{4 i} \Delta I N F_{t-i} \\
& +\sum_{i=0}^{n} \gamma_{5 i} \Delta G D S_{t-i}+\gamma_{6} G D C_{t-1}+\gamma_{7} B F A_{t-1} \\
& \Delta I N F_{t}=\delta_{0}+\sum_{i=1}^{n} \delta_{1 i} \Delta I N F_{t-i}+\sum_{i=0}^{n} \delta_{2 i} \Delta I N V_{t-i}+\sum_{i=0}^{n} \delta_{3 i} \Delta B F A_{t-i} \\
& +\sum_{i=0}^{n} \delta_{4 i} \Delta G D C_{t-i}+\sum_{i=0}^{n} \delta_{5 i} \Delta G D S_{t-i} \\
& +\delta_{6} B F A_{t-1}+\delta_{7} I N V_{t-1}+\delta_{8} I N F_{t-1} \\
& +\delta_{9} G D C_{t-1}+\delta_{10} G D S_{t-1}+\varepsilon_{5 t}
\end{aligned}
$$

The multivariate causality model is then presented as follows:

$$
\begin{aligned}
& \Delta I N V_{t}=\alpha_{0}+\sum_{i=1}^{n} \alpha_{1 i} \Delta I N V_{t-i}+\sum_{i=1}^{n} \alpha_{2 i} \Delta B F A_{t-i} \\
&+\sum_{i=1}^{n} \alpha_{3 i} \Delta I N F_{t-i}+\sum_{i=1}^{n} \alpha_{4 i} \Delta G D C_{t-i} \\
&+\sum_{i=1}^{n} \alpha_{5 i} \Delta G D S_{t-i}+\alpha_{6} E C T_{t-1}+\mu_{1 t} \\
& \Delta B F A_{t}=\beta_{0}+\sum_{i=1}^{n} \beta_{1 i} \Delta I N V_{t-i}+\sum_{i=1}^{n} \beta_{2 i} \Delta B F A_{t-i} \\
&+\sum_{i=1}^{n} \beta_{3 i} \Delta I N F_{t-i}+\sum_{i=1}^{n} \beta_{4 i} \Delta G D C_{t-i} \\
&+\sum_{i=1}^{n} \beta_{5 i} \Delta G D S_{t-i}+\beta_{6} E C T_{t-1}+\mu_{2 t} \\
& \Delta G D S_{t}=\rho_{0}+\sum_{i=1}^{n} \rho_{1 i} \Delta I N V_{t-i}+\sum_{i=1}^{n} \rho_{2 i} \Delta B F A_{t-i} \\
&+\sum_{i=1}^{n} \rho_{3 i} \Delta I N F_{t-i}+\sum_{i=1}^{n} \rho_{4 i} \Delta G D C_{t-i} \\
&+\sum_{i=1}^{n} \rho_{5 i} \Delta G D S_{t-i}+\rho_{6} E C T_{t-1}+\mu_{3 t}
\end{aligned}
$$

\section{EMPIRICAL RESULTS}

Since the ARDL bounds test works only with variables integrated to a maximum order of 1, the augmented DickeyFuller generalised least square and the Perron (1997) PPURoot unit root tests were employed. Unit Root test results are reported in Table 2.

All the variables under consideration are at most integrated of order 1 . Therefore, given the confirmation of the order of integration to be at most 1 , the next step is to test the possibility of cointegration among the variables using the 
Table 1. Unit Root Test Results

\begin{tabular}{|c|c|c|c|c|c|c|c|c|}
\hline \multicolumn{5}{|c|}{ DICKEY-FULLER GENERALISED LEAST SQUARE (DF-GLS) } & \multicolumn{4}{|c|}{ PERRON (1997) UNIT ROOT TEST (PPUROOT) } \\
\hline \multirow{2}{*}{ Variable } & \multicolumn{2}{|c|}{ Levels - I(0) } & \multicolumn{2}{|c|}{ Differences - I(1) } & \multicolumn{2}{|c|}{ Levels - I(0) } & \multicolumn{2}{|c|}{ Differences - I(1) } \\
\hline & No trend & With trend & No trend & With trend & No trend & With trend & No trend & With trend \\
\hline$I N V$ & $-2.086 * *$ & $-3.169^{* * *}$ & & & $-6.57 * * *$ & $-6.54^{* * * *}$ & - & - \\
\hline$L L$ & -1.026 & -1.358 & $-7.29 * * * *$ & $-7.79 * * *$ & -4.06 & -4.443 & $-8.25 * * * *$ & $-8.30 * * * *$ \\
\hline$D C F S$ & $-1.821^{*}$ & -1.883 & $-5.74 * * * *$ & $-5.84^{* * * *}$ & -3.68 & -3.001 & $-6.47^{* * * * *}$ & $-8.45^{* * * *}$ \\
\hline$D C P S$ & -1.387 & -1.463 & $-6.29 * * * *$ & $-6.35 \% * *$ & -2.47 & -3.097 & $-9.11 * * *$ & $-9.39 * * * *$ \\
\hline$F D I$ & -1.623 & -1.684 & $-6.30^{* * * * *}$ & $-6.45^{* * * * *}$ & -2.14 & -2.967 & $-7.99^{* * * * *}$ & $-8.25^{* * * *}$ \\
\hline$I N F$ & $-7.6^{\text {***** }}$ & $-7.69 * * * *$ & & & -8.75 ***** & $-8.83 * * * *$ & & \\
\hline$G D C$ & -1.96 & -2.131 & $-4.17^{* * * * * *}$ & $-4.20 * * * *$ & -3.949 & -4.392 & $-6.08^{* * * *}$ & $-6.24 * * *$ \\
\hline GDS & -1.32 & $-3.43^{* * *}$ & -4.77 **** & $-6.11 * * *$ & -4.523 & -5.148 & $-5.771 * *$ & $-5.763 * *$ \\
\hline
\end{tabular}

Note: $* * *$ and $* * *$ denote stationarity at the $10 \%, 5 \%$ and $1 \%$ significance levels, respectively

Table 2. Bounds F-Test for Cointegration

\begin{tabular}{|c|c|c|c|c|c|c|c|}
\hline \multicolumn{4}{|c|}{$\begin{array}{l}\text { Model A: Investment (INV), Liquid Liabilities (LL), GDP per Capita } \\
\text { growth (GDC), Savings (GDS) and Inflation (INF) }\end{array}$} & \multicolumn{4}{|c|}{$\begin{array}{l}\text { Model B: Investment (INV), Domestic credit provided by } \\
\text { financial sector (DCFS), GDP per Capita growth (GDC), Savings } \\
\text { (GDS) and Inflation (INF) }\end{array}$} \\
\hline $\begin{array}{c}\text { Dependent } \\
\text { Variable }\end{array}$ & Function & F-statistic & $\begin{array}{c}\text { Cointegration } \\
\text { Status } \\
\end{array}$ & $\begin{array}{c}\text { Dependent } \\
\text { Variable }\end{array}$ & Function & F-statistic & $\begin{array}{c}\text { Cointegration } \\
\text { Status }\end{array}$ \\
\hline INV & $\begin{array}{c}\text { F(INV|LL,GDC, } \\
\text { GDS,INF) }\end{array}$ & $4.4459 \% *$ & Cointegrated & INV & $\begin{array}{c}\text { F(INV|DCFS,GDC, } \\
\text { GDS,INF) }\end{array}$ & 3.3781 & Not Cointegrated \\
\hline LL & $\begin{array}{l}\text { F(LL|INV,GDC, } \\
\text { GDS,INF) }\end{array}$ & 0.71249 & Not Cointegrated & DCFS & $\begin{array}{c}\text { F(DCFS|INV,GDC, } \\
\text { GDS,INF) }\end{array}$ & 2.8535 & Not Cointegrated \\
\hline GDC & $\begin{array}{l}\text { F(GDC|LL,INV, } \\
\text { GDS,INF) }\end{array}$ & 1.2490 & Not Cointegrated & GDC & $\begin{array}{c}\text { F(GDC|DCFS,INV, } \\
\text { GDS,INF) }\end{array}$ & 1.1939 & Not Cointegrated \\
\hline GDS & $\begin{array}{l}\text { F(GDS|LL,GDC, } \\
\text { INV,INF) }\end{array}$ & 1.3352 & Not Cointegrated & GDS & $\begin{array}{c}\text { F(GDS|DCFS,GDC, } \\
\text { INV,INF) }\end{array}$ & 1.2933 & Not Cointegrated \\
\hline INF & $\begin{array}{l}\text { F(INF|LL,GDC, } \\
\text { GDS,INV) }\end{array}$ & $6.9964^{* * * * *}$ & Cointegrated & INF & $\begin{array}{l}\text { F(INF|DCFS,GDC, } \\
\text { GDS,INV) }\end{array}$ & $6.4384^{* * * * *}$ & Cointegrated \\
\hline
\end{tabular}

Model C: Investment (INV), Domestic credit to private sector $\quad$ Model D: Investment (INV), Financial Development Index (FDI),
(DCPS), GDP per Capita growth (GDC), Savings (GDS) and
Inflation (INF)

\begin{tabular}{|c|c|c|c|c|c|c|c|}
\hline $\begin{array}{c}\text { Dependent } \\
\text { Variable }\end{array}$ & Function & F-statistic & $\begin{array}{c}\text { Cointegration } \\
\text { Status } \\
\end{array}$ & $\begin{array}{c}\text { Dependent } \\
\text { Variable }\end{array}$ & Function & F-statistic & $\begin{array}{c}\text { Cointegration } \\
\text { Status } \\
\end{array}$ \\
\hline INV & $\begin{array}{l}\text { F(INV|DCPS,GDC, } \\
\text { GDS,INF) }\end{array}$ & $3.6160 *$ & Cointegrated & INV & $\begin{array}{l}\text { F(INV|FDI,GDC, } \\
\text { GDS,INF) }\end{array}$ & $3.6182 *$ & Cointegrated \\
\hline DCPS & $\begin{array}{l}\text { F(DCPS|INV,GDC, } \\
\text { GDS,INF) }\end{array}$ & 1.7927 & Not Cointegrated & FDI & $\begin{array}{l}\text { F(FDI|INV,GDC, } \\
\text { GDS,INF) }\end{array}$ & 2.0433 & Not Cointegrated \\
\hline GDC & $\begin{array}{l}\text { F(GDC|DCPS,INV, } \\
\text { GDS,INF) }\end{array}$ & 1.3836 & Not Cointegrated & GDC & $\begin{array}{l}\text { F(GDC|FDI,INV, } \\
\text { GDS,INF) }\end{array}$ & 1.3793 & Not Cointegrated \\
\hline GDS & $\begin{array}{l}\text { F(GDS|DCPS,GDC, } \\
\text { INV,INF) }\end{array}$ & 2.2366 & Not Cointegrated & GDS & $\begin{array}{l}\text { F(GDS|FDI,GDC, } \\
\text { INV,INF) }\end{array}$ & 1.2145 & Not Cointegrated \\
\hline INF & $\begin{array}{c}\text { F(INF|DCPS,GDC, } \\
\text { GDS,INV) }\end{array}$ & $6.1344^{* * * *}$ & Cointegrated & INF & $\begin{array}{l}\text { F(INF|FDI,GDC, } \\
\text { GDS,INV) }\end{array}$ & $6.3370 * * *$ & Cointegrated \\
\hline \multicolumn{8}{|c|}{ Asymptotic Critical Values } \\
\hline \multirow{3}{*}{\multicolumn{2}{|c|}{$\begin{array}{c}\text { Pesaran et al 2001:300 Table CI(iii) Case } \\
\text { III }\end{array}$}} & \multicolumn{2}{|r|}{$1 \%$} & \multicolumn{2}{|r|}{$5 \%$} & \multicolumn{2}{|r|}{$10 \%$} \\
\hline & & $\mathrm{I}(0)$ & $\mathrm{I}(1)$ & $\mathrm{I}(0)$ & $\mathrm{I}(1)$ & $\mathrm{I}(0)$ & $\mathrm{I}(1)$ \\
\hline & & 3.74 & 5.06 & 2.86 & 4.01 & 2.45 & 3.52 \\
\hline
\end{tabular}

Note: *, ** and ${ }^{* * * *}$ denotes significance at the $10 \%, 5 \%$ and $1 \%$ significance levels, respectively

ARDL bounds testing procedure. To establish if there is cointegration in the variables under study, the bounds F-test is employed. If there is cointegration, the estimated causality model would contain the error correction term as one of the regressors, and the opposite would also be true (i.e., no cointegration, no error correction term in the estimated model). The empirical results of the ARDL bounds F-test for both bank-based financial development (Model A) and marketbased financial development (Model B) are given in Table 3.

Results show that, for Model A, there is short-run bidirectional causality between liquid liabilities and investment and a long-run unidirectional causality relationship from liquid liabilities to investment. Other results for Model A show that liquid liabilities Granger cause inflation both in the short run and in the long run; liquid liabilities also Granger cause savings in the short run, and savings Granger cause economic growth.

For Model B, the bulk of the results are for short-run relationships, that is, domestic credit provided by the financial sector precedes investment while investment precedes economic growth. Other short-run results show that inflation precedes domestic credit provided by the financial sector and savings; inflation precedes savings and economic growth 
Table 3. Granger-Causality Test Results

\begin{tabular}{|c|c|c|c|c|c|c|c|c|c|c|c|c|c|}
\hline \multicolumn{7}{|c|}{$\begin{array}{l}\text { Model A: Investment (INV), Liquid Liabilities (LL), GDP per Capita } \\
\text { growth (GDC), Savings (GDS) and Inflation (INF) }\end{array}$} & \multicolumn{7}{|c|}{$\begin{array}{l}\text { Model B: Investment (INV), Domestic credit provided by } \\
\text { financial sector (DCFS), GDP per Capita growth (GDC), Savings } \\
\text { (GDS) and Inflation (INF) }\end{array}$} \\
\hline \multirow{2}{*}{$\begin{array}{l}\text { Dependent } \\
\text { Variable }\end{array}$} & \multicolumn{5}{|c|}{ F-statistics (probability) } & \multirow{2}{*}{$\begin{array}{c}E C T_{t} \\
{[\mathrm{t}-} \\
\text { statistics] }\end{array}$} & \multirow{2}{*}{$\begin{array}{l}\text { Dependent } \\
\text { Variable }\end{array}$} & \multicolumn{5}{|c|}{ F-statistics (probability) } & \multirow{2}{*}{$\begin{array}{c}E C T_{t} \\
{[\mathrm{t}-} \\
\text { statistics] }\end{array}$} \\
\hline & $\Delta I N V_{t}$ & $\Delta L L_{t}$ & $\Delta G D C_{t}$ & $\Delta G D S_{t}$ & $\Delta I N F_{t}$ & & & $\Delta I N V_{t}$ & $\triangle D C F S_{t}$ & $\triangle G D C_{t}$ & $\Delta G D S_{t}$ & $\Delta I N F_{t}$ & \\
\hline$\Delta I N V_{t}$ & - & & & & & & $\Delta I N V_{t}$ & & & & & & - \\
\hline$\Delta L L_{t}$ & & - & & & & - & $\triangle D C F S_{t}$ & $(0.7$ & - & & & & - \\
\hline$\Delta G D C_{t}$ & & & - & & & - & $\Delta G D C_{t}$ & $\begin{array}{l}3.1527^{*} \\
(0.89)\end{array}$ & $\begin{array}{c}0.26561 \\
(0.768) \\
\end{array}$ & - & & & - \\
\hline$\Delta G D S_{t}$ & $\begin{array}{c}0.28749 \\
(0.752) \\
\end{array}$ & $\begin{array}{l}7.22^{* * * * *} \\
(0.002)\end{array}$ & $\begin{array}{c}0.44907 \\
(0.641) \\
\end{array}$ & - & $\begin{array}{c}0.70169 \\
(0.501) \\
\end{array}$ & & $\Delta G D S_{t}$ & $\begin{array}{c}0.15656 \\
(0.855) \\
\end{array}$ & $\begin{array}{l}1.2442 \\
(0.270)\end{array}$ & $\begin{array}{c}0.14127 \\
(0.569) \\
\end{array}$ & - & & - \\
\hline$\Delta I N F_{t}$ & $\begin{array}{c}0.00972 \\
(0.990)\end{array}$ & $\begin{array}{l}3.291 * \\
(0.082)\end{array}$ & $\begin{array}{c}0.15816 \\
(0.854)\end{array}$ & $\begin{array}{c}0.36967 \\
(0.693)\end{array}$ & - & $\begin{array}{c}-0.6538 * * * \\
{[-5.5273)}\end{array}$ & $\Delta I N F_{t}$ & $\begin{array}{c}0.21660 \\
(0.644)\end{array}$ & $\begin{array}{l}1.3549 \\
(0.269)\end{array}$ & $\begin{array}{c}3.3743^{*} \\
(0.073)\end{array}$ & $\begin{array}{c}0.12975 \\
(0.879)\end{array}$ & - & $\begin{array}{c}-0.7534^{* * *} \\
{[-3.1922]}\end{array}$ \\
\hline
\end{tabular}

Model C: Investment (INV), Domestic credit to private sector (DCPS), GDP per Capita growth (GDC), Savings (GDS) and Inflation (INF)

\begin{tabular}{|c|c|c|c|c|c|c|c|c|c|c|c|c|c|}
\hline \multirow{2}{*}{$\begin{array}{c}\text { Dependent } \\
\text { Variable }\end{array}$} & \multicolumn{5}{|c|}{ F-statistics (probability) } & \multirow{2}{*}{$\begin{array}{c}E C T_{t} \\
{[\mathrm{t}-} \\
\text { statistics] }\end{array}$} & \multirow{2}{*}{$\begin{array}{c}\text { Dependent } \\
\text { Variable }\end{array}$} & \multicolumn{5}{|c|}{ F-statistics (probability) } & \multirow{2}{*}{$\begin{array}{c}E C T_{t} \\
{[t-} \\
\text { statistics] }\end{array}$} \\
\hline & $\Delta I N V_{t}$ & $\triangle D C P S_{t}$ & $\Delta G D C_{t}$ & $\Delta G D S_{t}$ & $\Delta I N F_{t}$ & & & $\Delta I N V_{t}$ & $\Delta F D I_{t}$ & $\Delta G D C_{t}$ & $\Delta G D S_{t}$ & $\Delta I N F_{t}$ & \\
\hline$\Delta I N V_{t}$ & - & $\begin{array}{l}1.7543 \\
(0.192)\end{array}$ & $\begin{array}{l}5.551^{* * *} \\
(0.027)\end{array}$ & & & $\begin{array}{c}-0.654^{* * * * *} \\
{[4.1916]}\end{array}$ & $\Delta I N V_{t}$ & - & $\begin{array}{l}2.9637^{*} \\
(0.062)\end{array}$ & $\begin{array}{c}0.32239 \\
(0.726) \\
\end{array}$ & & $\begin{array}{c}0.97399 \\
(0.386) \\
\end{array}$ & $\begin{array}{l}-0.4484^{* * *} \\
{[-2.9781]}\end{array}$ \\
\hline$\triangle D C P S_{t}$ & $\begin{array}{l}1.5065 \\
(0.233) \\
\end{array}$ & - & $\begin{array}{l}3.976^{\text {***** }} \\
(0.026)\end{array}$ & $\begin{array}{l}2.1974 \\
(0.123)\end{array}$ & $\begin{array}{l}1.1534 \\
(0.325)\end{array}$ & $\mathrm{L}-\mathrm{s}-\mathrm{J}$ & $\Delta F D I_{t}$ & $\begin{array}{c}0.87294 \\
(0.425)\end{array}$ & I & $\begin{array}{l}2.960^{*} \\
(0.098)\end{array}$ & $\begin{array}{l}3.710^{*} \\
(0.066)\end{array}$ & $\begin{array}{c}0.94490 \\
(0.396)\end{array}$ & \\
\hline$\Delta G D C_{t}$ & $\begin{array}{l}3.134^{*} \\
(0.09)\end{array}$ & $\begin{array}{c}0.74778 \\
(0.478) \\
\end{array}$ & - & $\begin{array}{l}1.5660 \\
(0.220) \\
\end{array}$ & $\begin{array}{c}0.61351 \\
(0.546)\end{array}$ & - & $\Delta G D C_{t}$ & $\begin{array}{l}3.366^{* * * *} \\
(0.048)\end{array}$ & $\begin{array}{c}0.33618 \\
(0.716) \\
\end{array}$ & - & $\begin{array}{l}1.1106 \\
(0.338)\end{array}$ & $\begin{array}{c}0.52789 \\
(0.594) \\
\end{array}$ & - \\
\hline$\Delta G D S_{t}$ & $\begin{array}{c}0.73571 \\
(0.485)\end{array}$ & $\begin{array}{c}0.46759 \\
(0.630)\end{array}$ & $\begin{array}{c}0.28143 \\
(0.756)\end{array}$ & - & $\begin{array}{l}3.398^{*} \\
(0.078)\end{array}$ & - & $\Delta G D S_{t}$ & $\begin{array}{c}0.25518 \\
(0.796)\end{array}$ & $\begin{array}{c}0.52464 \\
(0.595)\end{array}$ & $\begin{array}{c}0.14423 \\
(0.866)\end{array}$ & - & $\begin{array}{l}2.852^{* * *} \\
(0.041)\end{array}$ & \\
\hline$\Delta I N F_{t}$ & $\begin{array}{c}0.33779 \\
(0.564) \\
\end{array}$ & $\begin{array}{l}3.735^{*} \\
(0.065)\end{array}$ & $\begin{array}{c}0.15573 \\
(0.695)\end{array}$ & $\begin{array}{l}0.0939 \\
(0.760) \\
\end{array}$ & - & $\begin{array}{l}-0.760^{\text {******** }} \\
{[-3.3709]}\end{array}$ & $\Delta I N F_{t}$ & $\begin{array}{c}0.17368 \\
(0.841)\end{array}$ & $\begin{array}{l}3.0482 * \\
(0.058)\end{array}$ & $\begin{array}{c}0.29223 \\
(0.748)\end{array}$ & $\begin{array}{c}0.02163 \\
(0.979)\end{array}$ & - & $\begin{array}{l}-0.19185 \\
{[-3.1124]}\end{array}$ \\
\hline
\end{tabular}

Note: * ** and **** denotes significance at the $10 \%, 5 \%$ and $1 \%$ significance levels, respectively

precedes inflation. In addition, there is long-run unidirectional causality from economic growth to inflation.

For Model C, results show that there is a short-run bidirectional causality relationship between economic growth and investment and a long-run unidirectional relationship from economic growth to investment. Further, economic growth Granger causes domestic credit to the private sector in the short run. Other results from Model 3 show that inflation Granger causes savings and domestic credit to private sector Granger causes inflation, both in the short run. In addition, there is a long-run relationship from domestic credit to private sector to inflation.

Deductions from Model D show only short-run causality relationships. Of note, financial development is found to Granger cause investment, economic growth Granger causes financial development while investment Granger causes economic growth. Other results from Model D show that there is unidirectional causality from savings to financial development, from inflation to savings and from financial development to inflation.

Therefore, the finance-investment-growth causal relationship in the Republic of Congo is highly dependent on the choice of the financial development indicator used. Notable though is the deduction that all financial development indicators except domestic credit to the private sector Granger cause investment. In addition, investment has been found to Granger cause economic growth in the short run when domestic credit provided by the financial sector and the composite financial development index are included as measures of financial development in subsequent models. More so, when the composite financial development indicator is used to measure financial development, there is a 'looped' finance-investment-growth causal relationship. That is, financial development precedes investment, investment precedes economic growth and economic growth inherently precedes financial development.

\section{CONCLUSION}

The finance-investment-growth causal relationship in the Republic of Congo has been empirically examined for the period of 1960 to 2017. The causal relationship between financial development, investment and economic growth was examined using four multivariate Granger-causality models. The savings and inflation ratios were included as intermitting variables in order to address the problem of omission-ofvariable bias. The study found that the finance-investmentgrowth causal relationship in the Republic of Congo is highly dependent on the choice of the financial development indicator used. In addition, all financial development indicators, except domestic credit to the private sector, were found to Granger cause investment in the short run. Furthermore, investment has been found to Granger cause economic growth, in the short run, when domestic credit 
provided by the financial sector and the composite financial development index are included as measures of financial development in subsequent models. Moreover, when the composite financial development indicator is used to measure financial development, there is a 'looped' finance-investmentgrowth causal relationship. That is, financial development precedes investment, investment precedes economic growth and economic growth inherently precedes financial development. Therefore, dependent on the policy focus, the management of the economy through financial development should take into account the financial indicator so as to predict and/or achieve the resultant policy objectives.

\section{REFERENCES}

Abu-Bader, S. and Abu-Qarn, A.S. (2008). Financial development and economic growth, the Egyptian experience. Journal of Policy Modeling, 30, 887-898. https://doi.org/10.1016/j.jpolmod.2007.02.001

Akinlo, A. E. and Egbetunde, T. (2010). Financial development and economic growth: the experience of 10 sub-Saharan African countries revisited. The Review of Finance and Banking, 2(1), 17-28.

Ang, J. B. and McKibbin, W. J. (2007). Financial liberalisation, financial sector development and growth: evidence from Malaysia. Journal of Development Economics, 84(1), 215-233. https://doi.org/10.1016/j.jdeveco.2006.11.006

Asongu, S. (2014). Linkages between investment flows and financial development: Causality evidence from selected African countries. African Journal of Economic and Management Studies, 5(3), 269-299. https://doi.org/10.1108/AJEMS-05-2012-0036

BEAC (2019). Available at: https://www.beac.int (Accessed: 30 May 2019).

Beck, T., Levine, R. and Loayza, N. (2000). Finance and the sources of growth. Journal of Financial Economics, 58(1/2), 261-300. https://doi.org/10.1016/S0304-405X(00)00072-6

Berthelemy, J. C. and Varoudakis, A. (1996). Economic growth, convergence clubs, and the role of financial development. Oxford Economic Papers, 48(2), 300-328. https://doi.org/ 10.1093/oxfordjournals.oep.a028570

Caporale, G. M., Howells, P. and Soliman, A. M. (2005). Endogenous growth models and stock market development: evidence from four countries. Review of Development Economics, 9(2), 166-176. https://doi.org/ 10.1111/j.1467-9361.2005.00270.x

Carp, L. (2012). Can stock market development boost economic growth? Empirical evidence from emerging markets in Central and Eastern Europe. Procedia Economics and Finance, 3, 438-444. https://doi.org/10.1016/S22125671(12)00177-3

Chaudhry, I. S. (2007). Financial Liberalization and Macroeconomic Performance: Empirical Evidence from Pakistan. [Online] Available at: http://www.gredeg. cnrs.fr/colloques/nfi/papers/PapierOnLine/Chaudry.pdf

Clark, J. (1997). Petro-politics in Congo. Journal of Democracy, 8(3), 62-76. https://doi.org/10.1353/jod.1997.0038
Clark, J. F. (2002). The neo - colonial context of the democratic experiment of Congo - Brazzaville. African Affairs 101(403), 171-192. https://doi.org/10.1093/afraf/101.403. 171

Decalo, S. (1990). Coups and Army Rule in Africa: Studies in Military Style (2nd edn.). New Haven, Yale University Press.

Fase, M. M. G. and Abma, R. C. N. (2003). Financial environment and economic growth in selected Asian countries. Journal of Asian Economics, 14(1), 11-21. https://doi.org/10.1016/S1049-0078(02)00237-3

Ghali, K. H. (1999). Financial development and economic growth: the Tunisian experience. Review of Development Economics, 3(3), 310-322. https://doi.org/10.1111/14679361.00070

Huang, Y. (2011). Private investment and financial development in a globalized world. Empirical Economics, 41(1), 43-56. https://doi.org/10.1007/s00181-010-0394-3

Hussain, F. and Chakraborty, D. K. (2012). Causality between financial development and economic growth: evidence from an Indian State. The Romanian Economic Journal, 15(45), 27-48.

Jalilian, H. and Kirkpatrick, C. (2002). Financial development and poverty reduction in developing countries. International Journal of Finance and Economics, 7(2), 97-108. https://doi.org/10.1002/ijfe.179

Jung, W. S. (1986). Financial development and economic growth: international evidence. Economic Development and Cultural Change, 34(2), 333-346. https://doi.org/ 10.1086/451531

King, R. G. and Levine, R. (1993). Finance, entrepreneurship, and growth: theory and evidence. Journal of Monetary Economics, 32(3), 513-542. https://doi.org/10.1016/03043932(93)90028-E

Levine, R. (1997). Financial Development and Economic Growth: Views and Agenda. Journal of Economic Literature, 35, 688-726.

Levine, R. (2005). Finance and growth: theory and evidence. Handbook of economic growth, 1, 865-934. https://doi.org/ 10.1016/S1574-0684(05)01012-9

Levine, R., Loayza, N. and Beck, T. (2000). Financial intermediation and growth: causality and causes. Journal of Monetary Economics, 46(1), 31-77. https://doi.org/ 10.1016/S0304-3932(00)00017-9

Luintel, K. B. and Khan, M. (1999). A quantitative reassessment of the finance growth nexus: evidence from a multivariate VAR. Journal of Development Economics, 60(2), 381-405. https://doi.org/10.1016/S0304-3878(99)00045-0

Majid, M. S. A. (2008). Does financial development matter for economic growth in Malaysia? An ARDL bound testing approach. Journal of economic Cooperation, 29(1), 61-82.

McKinnon, R. I. (1973). Money and Capital in Economic Development, Washington, DC: Brookings Institution.

Muyambiri, B. and Odhiambo, N. M. (2018). The causal relationship between financial development and investment: review of related empirical literature. Comparative Economic Research, 21(2), 119-136. https://doi.org/10.2478/cer-2018-0015 
Neusser, K. and Kugler, M. (1998). Manufacturing growth and financial development: evidence from OECD countries. Review of Economics and Statistics, 80, 638-646. https://doi.org/10.1162/003465398557726

Nyasha, S. and Odhiambo, N. M. (2015). Banks, stock market development and economic growth in South Africa: a multivariate causal linkage. Applied Economics Letters, 22(18), 1480-1485. https://doi.org/10.1080/13504851. 2015.1042132

Odedokun, M. O. (1996). Financial policy and efficiency of resource utilization in developing countries. Growth and Change, 27(3), 269-297. https://doi.org/10.1111/j.14682257.1996.tb00906.x

Odhiambo, N. M. (2004). Is financial development still a spur to economic growth? A causal evidence from South Africa. Savings and Development, 28, 47-62.

Odhiambo, N. M. (2008) Financial development in Kenya: a dynamic test of the finance-led growth hypothesis. Economic Issues, 13(2), 21-36.

Odhiambo, N. M. (2010). Finance-investment-growth nexus in South Africa: an ARDL-bounds testing procedure. Economic Change and Restructuring, 43, 205-219. https://doi.org/10.1007/s10644-010-9085-5

Perron, P. (1997). Further evidence on breaking trend functions in macroeconomic variables. Journal of econometrics, 80 (2), 355-385. https://doi.org/10.1016/ S0304-4076(97)00049-3
Pesaran, M. H., Shin, Y. and Smith, R. (2001). Bound testing approaches to the analysis of level relationship. Journal of Applied Econometrics, 16(3), 289-326. https://doi.org/ 10.1002/jae.616

Rachdi, H. and Mbarek, B. (2011) The causality between financial development and economic growth: panel data cointegration and GMM system approaches. International Journal of Economics and Finance, 3, 143-151. https://doi.org/10.5539/ijef.v3n1p143

Rousseau, L. and Wachtel, P. (1998). Financial intermediation and economic performance: historical evidence from five industrialised countries. Journal of Money, Credit and Banking, 30, 657-678. https://doi.org/10.2307/2601123

Shan, J. and Jianhong, Q. (2006). Does Financial Development Lead Economic Growth? The Case of China. Annals of Economics and Finance, 7, 197-212.

Shan, J. and Morris, A., (2002). Does financial development 'lead' economic growth?'. International Review of Applied Economics, 16(2), 153-168. https://doi.org/10.1080/ 02692170110118885

Shaw, E. S. (1973). Financial Deepening in Economic Development. New York: Oxford University Press.

World Bank. (2019). World Development Indicators (WDI), Washington, D.C.: World Bank. http://data.worldbank.org/ data-catalog/world-development-indicators (Accessed: 22 October 2019).

$\mathrm{Xu}$, Z. (2000). Financial development, investment, and economic growth. Economic Inquiry, 38(2), 331-344. https://doi.org/10.1111/j.1465-7295.2000.tb00021.x 